Anastasia Telaak, Danzig

\title{
Geteilte Erinnerung. Galizien in Sabrina Janeschs „Katzenberge“ und Jenny Erpenbecks „Aller Tage Abend“
}

\begin{abstract}
Der vorliegende Beitrag ist eine Lektüre der Romane von Sabrina Janesch und Jenny Erpenbeck mit Blick auf das darin gestaltete Galizien, mittelosteuropäischer Kultur- und Gedächtnisort und literarischer Topos zugleich. Im Zentrum der Lektüre stehen die unterschiedlichen narrativen und semiotischen Modi der Darstellung von interkulturellen Konstellationen, intergenerationellen Überlieferungsmustern und Zugehörigkeitskonstruktionen.
\end{abstract}

„Ostgalizische Erde ist verschwenderisch und reich. Sie hat fettes Öl, gelben Tabak, bleischweres Getreide, alte verträumte Wälder und Seen und vor allem schöne, gesunde Menschen: Ukrainer, Polen, Juden. Alle drei sehen sich ähnlich, trotz verschiedener Sitten und Gebräuche. [...]“1

Im Roman „Da geht ein Mensch. Roman eines Lebens“ nennt der 1890 in Werbowitz (ehemals Galizien, heute Ukraine) in einer jüdischen Bauernfamilie geborene Schauspieler Alexander Granach die drei größten ethnisch-kulturellen Gruppen im historischen Galizien beinah in einem Atemzug. Wie sich ihre Lebenswirklichkeit gestaltet, darüber sagt das Zitat nichts, allenfalls behauptet es eine Ähnlichkeit im idealisierten Aussehen, die man - kulturellen Unterschieden zum Trotz, und vor der in romantischer Üppigkeit gezeichneten Landschaft als Voraussetzung für eine harmonische Koexistenz nehmen könnte.

Um die galizischen Erfahrungswelten von Polen und Juden, insbesondere um jüdischpolnische und polnisch-ukrainische Beziehungen in Galizien, die das Bild im eben gelesenen Zitat in anderem Licht zeigen, geht es in zwei neueren deutschsprachigen Romanen: „Katzenberge“ von Sabrina Janesch (2010), einer 1985 in Westdeutschland geborenen, dann im Westen Deutschlands aufgewachsenen deutsch-polnischen Schriftstellerin, und „Aller Tage Abend“ von Jenny Erpenbeck (2012), geboren 1967 und aufgewachsen in der DDR. Beide gestalten Galizien als komplexen Geschichts- und Gedächtnisraum. Als imaginärer Bezugspunkt im Kontext der postmémoire/postmemory erscheint die historische Landschaft als ein Palimpsest traumatischen Charakters; von hier aus leisten beide Autorinnen eine kritische Literarisierung kommunikativer und kollektiver Gedächtnisspuren in einem intergenerationellen Kontext sowie damit zusammenhängender Konstruktionen von (Nicht-)

\footnotetext{
${ }^{1}$ Alexander Granach, Da geht ein Mensch. Roman eines Lebens. München/Zürich 1990, 1, hier zit. nach Heiko Haumann, Geschichte der Ostjuden, 19995 169.
} 
Zugehörigkeit. Der Topos Galizien ist in beiden Romanen allerdings unterschiedlich strukturiert; sowohl in interkulturellen Konstellationen, als auch im kommunikativen Gedächtnis kommt ihm eine jeweils unterschiedliche Wertigkeit zu. Wie- so soll im Folgenden erörtert werden - äußert sich dies narrativ und semiotisch? Was wird jeweils in den Mittelpunkt gerückt und in welchem Modus? Welche Zeichen zirkulieren im Text und produzieren dabei Sinn - oder auch nicht? Schließlich: Womit hängt der unterschiedliche Fokus auf Galizien sowie die unterschiedliche Zirkulation und Produktivität bestimmter Zeichen zusammen?

In dieser Hinsicht muss zunächst dem jeweils verschiedenen zeitlichen Rahmen Rechnung getragen werden, in dem die Narrative situiert sind: Bei Janesch reicht die erzählte/erinnerte Zeit von ca. 1920-1944, da Galizien - mit Ausnahme seiner vorübergehenden Eingliederung ins nationalsozialistische Generalgouvernement (1941-1944) - Teil des polnischen Staates war, und verschränkt sich, von wenigen auf die 80er-/90er-Jahre bezogenen Episoden abgesehen, mit der ersten Dekade des 21. Jahrhunderts, d.h. geographisch mit der heutigen Ukraine. Bei Erpenbeck bezieht sich das erzählte Galizien im Kern auf die Jahre 19021918, also auf das Kronland Galizien und Lodomerien als Teil der Habsburgermonarchie.

\section{Bergung des Mythos}

In „Katzenberge“, zwischen fiktiver Biographie, Reise- und Entwicklungsroman angesiedelt, nimmt Nele Leibert, Tochter einer polnischen Mutter und eines deutschen Vaters, den Tod des polnischen Großvaters Janeczko zum Anlass, von Berlin, ihrem Wohnort, nach Niederschlesien zu reisen. Erinnerungen an die beim vertrauten „djadjo“ verbrachten Ferien in der Kindheit, Andeutungen der polnischen Verwandten über ein düsteres Kapitel in seiner Vergangenheit und schließlich die von der Mutter aufgeworfene Frage nach der „eigentlichen“ Herkunft führen im Folgenden dazu, dass sich die Protagonistin auf die Reise nach Zastavne im ehemals polnischen Galizien, heute ukrainisches Gebiet, macht, wo der Großvater einst geboren und aufgewachsen war. Dabei verschränkt Janesch zwei in der neueren Geschichte Polens und Deutschlands einschneidende Geschehnisse miteinander: zum einen die Erfahrung mörderischer Gewalt der in Wolhynien lebenden Polen durch die ukrainischen Bewohner und ihre Vertreibung zwischen 1943-1945; zum anderen die „Repatriierung“ der Vertriebenen bzw. Zwangsumgesiedelten im ehemals von den Nationalsozialisten besetzten Schlesien nach der Vertreibung von Deutschen daraus. ${ }^{2}$

\footnotetext{
${ }^{2}$ Vgl. hierzu „Galizien und Schlesien. Zwangsumsiedlung und Vertreibung in den vierziger Jahren. Galizien“ in: http://www.sabrinajanesch.de/werke/katzenberge/galizien-und-schlesien/ ; 31.10.13; außerdem „Der Konflikt mit den Ukrainern in Wolhynien und Ostgalizien“, in: Atlas Zwangsumsiedlung, Flucht und Vertreibung. Ostmitteleuropa 1939-1959. Warszawa 2009, 71-74, sowie Gerhard Gnauck: „Männer und Frauen, grausam mit
} 
„Großvater sagte, ...“: Diese durch den Roman hindurch wiederholte Formulierung bildet nicht zufällig das Leitmotiv des Romans: Das im Prozess des Erzählens sich vollziehende Erinnern an „Anfangsworte“, das Verweben und Neu-Weben bereits vorgefundener Geschichten, denen wiederum eine „identitätsprägende Bedeutung für die Zukunft“ zugeschrieben werden ${ }^{3}$, garantiert formal und inhaltlich das sinnstiftende, dabei notwendig Distanz anzeigende Netz, in dem die junge Ich-Erzählerin zu Beginn des 21. Jahrhunderts eine Selbstverortung zwischen Deutschland und Polen unternimmt. Der galizische Großvater - und mit ihm der Ort, wo er „geboren worden war, wo alles begonnen hatte (...) $)^{\text {4 }}$ - erweist sich mithin als ein Vor-Bild, über das sich die Ich-Erzählerin unmittelbar als dessen würdige Nachfahrin konfiguriert, nämlich als Schöpferin des von ihm, „dem eigentlichen Erzähler“ 5 , inspirierten Galizien-Romans, eines literarischen galizischen Kosmos. Diese Konstruktion gewährleistet ihrerseits zweierlei: Über das vom Großvater vermittelte, kommunikative Gedächtnis gelingt der Erzählerin, stellvertretend für die gesamte polnische Familie, zum einen die Befriedung eines von Galizien ausgehenden, tabuisierten Familientraumas von biblischen Ausmaßen: der Großvater, so wird schweigend unterstellt, habe den eigenen Bruder auf der Flucht aus Galizien ermordet, weil dieser mehr oder weniger mit den ukrainischen Anführern eines Pogroms an den polnischen Bewohnern von Zastavne kollaboriert habe. Über die Reflexion der vom Großvater vermittelten, dabei auch schon kollektiven Erinnerung an die traumatische Vertreibungserfahrung leistet die Erzählerin zum anderen die Befriedung des Dämons der Geschichte. Am Ende steht schließlich eine gelungene deutsch-polnische Autopoiesis, in deren Rahmen die bei den Vorfahren aufgefundenen Ansätze zu interkulturellen Beziehungsformen in ein transkulturelles Konzept überführt werden. ${ }^{6}$

\footnotetext{
Äxten zerhackt“, in: Die Welt, 26.06.2013; http://www.welt.de/geschichte/zweiter-weltkrieg/article117455158/ Maenner-und-Frauen-grausam-mit-Aexten-zerhackt.html; 08.01.2014.

${ }^{3}$ Vgl. Aleida Assmann zum Stichwort „Mythos“ in dies., Einführung in die Kulturwissenschaft. Grundbegriffe, Themen, Fragestellungen. Berlin 2008², 167. Insbesondere im Zusammenhang mit der Abwandlung der Schöpfungsgeschichte in den Erzählungen des Großvaters (s. weiter unten im Text) wird „Mythos“ als „narrative Kategorie“ verstanden, nämlich (in Anlehnung an Mircea Eliade) als „Erzählung über die Ursprünge“; vgl. Paul Ricoeur: „Poetik und Symbolik - Erfahrung, die zur Sprache kommt“, in: Friedrich Jaeger und Burkhard Liebsch (Hrsg.), Handbuch der Kulturwissenschaften Band 1: Grundlagen und Schlüsselbegriffe. Stuttgart und Weimar 2011, 93-105, hier 102; 96.

${ }^{4}$ Ebd., 96.

${ }^{5}$ Vgl. hierzu die „Danksagung“: „Besonders bedanken möchte ich mich [...] [B]ei meinem Großvater, dem eigentlichen Erzähler, für die Geschichten.“; ebd., 273.

${ }^{6}$ Zum Konzept der Transkulturalität und in diesem Zusammenhang zur Figur der „Transmigrantin“ - „nicht nur in einer, sondern in zwei Kulturen dauerhaft verankert“, sich hierdurch vom „,leidenden Heimatlosen“ unterscheidend - vgl. Dorothee Kimmich/Schamma Schahadat, Einleitung zu dies. (Hrsg.): Kulturen in Bewegung. Beiträge zur Theorie und Praxis der Transkulturalität. Bielefeld 2012, 7-21, hier 7; für das Zitat 14; außerdem Wolfgang Welsch: „Was ist eigentlich Transkulturalität?“, op. cit., 25-40.
} 
Wie wird „Galizien“ nun in den Erzählungen des Großvaters abgebildet? Und wie konfiguriert sich dieser Topos in der erzählerischen Vermittlung durch die der EnkelinErzählerin, so dass er die Kraft entfaltet, Zugehörigkeit und Identität zu stiften?

Zunächst spiegeln sich im erzählten Galizien des Großvaters Bilder eines kollektiven Gedächtnisses, die sich auf historisch Verbürgtes beziehen: vor allem die von Ukrainern an Polen verübten Massaker und das Elend der Vertreibung. Diese mnemonischen Präfigurationen treten allerdings mit zunehmender erzählerischer Entfernung von bewusst Erfahrenem hinter einer mythischen Perspektive auf das polnisch-bäuerliche Galizien zurück. Vermittelt durch die Enkelin-Erzählerin bzw. durch das leitmotivische „Großvater sagte, ...“ wird Galizien so zum zentralen Topos eines von der Autorin entwickelten, systematischen Verfahrens der Remythisierung, wird Galizien zum genuinen „Gedächtnisort“: ausgestattet mit einer „symbolischen Aura“, ohne „Referenten“ in der Wirklichkeit“, vielmehr zeichenhaft seinen „eigene[n] Referent[en]“ repräsentierend. ${ }^{7}$ In diesem auratisch-mythischen und re-mythisierten Netz zirkulieren zunächst Zeichen des Idyllischen, Bilder aus einem als ursprünglich gewähnten, vor der Vertreibung existierenden Zeit-Raums - jenem der Kindheit und einem noch vor ihr liegenden „illo tempore“8: Idyllisch nehmen sich die ausschließlich auf Polen und Ukrainer konzentrierten, interkulturellen Beziehungen aus: „[I]n Żdżary Wielkie lebten auf kleinstem Raum Polen und Ukrainer eng beieinander und sprachen beide Sprachen“ “9; bei der langwierigen Geburt des Großvaters singen alle Bauern samt Frauen „im Wechsel ukrainische und polnische Lieder“; die Mutter bringt dem kleinen Janeczko „anstelle des Vaterunsers die Namen unserer ukrainischen Nachbarn“ bei. Ebenso wirkmächtig sind die Zeichen eines zauberhaften Aberglaubens, die in den Geschichten des „djadjos“ zirkulieren, welche ihm schon „seine Großeltern erzählt hatten“10 - Geschichten über „Geister, Dämonen, Hexen und Waldfeen“, die die „Wälder und Flüsse Galiziens“ „bevölkern“. Galizien, so will die von der Erzählerin erinnerte, kindliche Phantasie, muss eine Welt sein, „in die man nicht so einfach gelangen konnte“ - ein so unheimlicher wie privilegierter Ort.

Eben dieses Element des Zauberisch-Abergläubischen, von der Erzählerin als hervorragendes Merkmal galizischer Polonität gedeutet, ist es auch, das eine subtile, durch verhaltene erzählerische Ironie bestimmte Grenze zwischen Re-Mythisierung und Entmythisierung markiert bzw. beides in der Schwebe hält. Augenfällig zeigt sich dies in der

\footnotetext{
${ }^{7}$ Pierre Nora, „Die Gedächtnisorte“ (1984), in: Stephan Günzel, Texte zur Theorie des Raums. Stuttgart 2013, 8489, hier 84; 88f.

${ }^{8}$ Ricoeur: „Poetik und Symbolik“, 102.

${ }^{9}$ Ebd., hier und für das im unmittelbar Folgenden Zitierte 254; 251;68.

${ }^{10}$ Ebd., hier und für das im unmittelbar Folgenden Zitierte 144; 71.
} 
Repräsentation Galiziens als paradiesischer „Anfang der Welt“11, der seine Wahrheit aus der Überzeugung des Großvaters bezieht, dass er „am richtigen Platz zur Welt gekommen war“, mit Menschen so besonderen Schlages wie er selbst: „Groß [...], ihre Haare von der gleichen Farbe wie der Weizen, der auf [...] endlosen Feldern gedieh; und obgleich Bauern, hatten sie ein ausgeprägtes Gefühl für Sprache und Kultur.“ Die herausragende Metapher, die in diesem (re-)mythisierten Raum zirkuliert, ist hier natürlich die 'satte und fette', die nährende „ölig schimmernd[e] galizische Erde“, der „czarnosiem“, zugleich Humus des Imaginären, aus dem der Roman hervorgeht: „Janeczko [...] war Teil seines Weizens, stimm- und reglose Wucherung des czarnoziem, der ölig schimmernden galizischen Schwarzerde.“

Die galizische Erde ist es auch, die am Ende in Form ihrer ironisch gebrochenen Apotheose die paradoxale ,Substanz‘ des Erinnerten/Erzählten bestätigt: Damit die endgültige Bannung des Familienfluchs ganz gelinge, so auch die Bannung des großen Dämons der Geschichte, legt die Enkelin nach ihrer Rückkehr eine im Kopftuch der Großmutter geborgene Handvoll davon heimlich auf dem Grab des Großvaters ab, oder vielmehr, was davon übriggeblieben ist: eine nahezu immaterielle, „haarfeine, schwarze Spur“. Galizien und das erzählte Wort im Roman - sie bilden also ein Paar, als solches ausgewiesen durch beider paradoxe, weil flüchtige Beständigkeit. Diese Spur reicht jedoch offenkundig, um den Roman mit der wörtlichen Wiedergabe jenes optimistischen Satzes zu beschließen, den der Großvater bei der Geburt seines zweiten Sohnes, des „ersten galizischen Schlesiers“, sagte - also um die einen aus dem Gedächtnis geborgenen Mythos zu vollenden, der - ungeachtet seiner Fiktionalität, oder vielleicht gerade kraft derselben? - Zugehörigkeit stiftet: „Der Himmel war/ist kornblumenblau, es würde/wird ein guter Tag werden.“12

\section{Blackbox Galizien}

Sabrina Janesch literarisiert Galizien aus einer Perspektive, die neben der Vertrautheit mit dem Verhältnis zwischen Deutschen und Polen und den damit verbundenen Diskursen eine Sensibilität für das spezifisch polnische Trauma der Vertreibung aus dem Osten am Ende des Zweiten Weltkriegs voraussetzt. Diese Affinität besitzt die 18 Jahre ältere Schriftstellerin Jenny Erpenbeck offenkundig nicht. Vielmehr lässt ihr zwei Jahre nach „Katzenberge“ publizierter

\footnotetext{
${ }^{11}$ Janesch, Katzenberge, hier und für die unmittelbar folgenden Zitate 264, 254, 253f., 238, 270.

12 Janesch, Katzenberge, 148, 272.
} 
Roman „Aller Tage Abend“ Bilder von und Diskurse über Galizien erkennen, die als prägend für ihre Generation und für deren Sozialisation in einem ausschließlich deutschen Kontext gelten können - im Falle Erpenbecks zunächst in Ostdeutschland, dann im vereinigten Deutschland. Dazu gehört zunächst die nahezu ausschließliche Verbindung Galiziens mit den jüdischen Lebenswelten, die dort vor dem Zweiten Weltkrieg und deren Vernichtung existierten, ein Umstand, der im (bis heute gültigen) Rang der Shoah als singulärem Gedächtnisort in der geschichtswissenschaftlichen Forschung, in dem, was als das kollektive/öffentliche Bewusstsein bezeichnet wird, sowie in der ungebrochenen Präsenz der Shoah in den Medien, einschließlich der Belletristik, seine Entsprechung findet. Für einen mit den einschlägigen Diskursen vertrauten und in Literatur bewanderten Leser verweist Galizien ferner in erster Linie auf Joseph Roth, außerdem auch auf Bruno Schulz: zwei Schriftsteller, die aus jüdischen Familien stammten, im Habsburgerreich, daher auch in stärkerem oder geringerem Maße mit deutscher Kultur und Sprache aufwuchsen, als Juden unmittelbar Opfer der nationalsozialistischen Verfolgungs- und Vernichtungspolitik wurden, und deren Werk maßgeblich im Hinblick auf die darin dargestellten, bedrohten jüdischen Lebenswelten im Kontext von Assimilation und Moderne rezipiert worden ist ${ }^{13}$.

Von den skizzierten Traditions- und Diskurslinien herkommend, mit ihnen zusammenhängende Mythen dabei auf eigene Weise bearbeitend knüpft Erpenbecks Roman an das habsburgisch-jüdische Galizien als einem der prominentesten Topoi in der deutschsprachigen Literatur an. Die Figur der Blackbox trägt hierbei dem Rechnung, was, neben anderen für Erpenbecks Galizien-Darstellung relevanten Figuren, als innerster Bedeutungskern des gesamten Romans ausgemacht werden kann: Wie eine Blackbox gewinnt das ostjüdische Galizien seine Bedeutung maßgeblich angesichts seines Zusammenbruchs, der Verwirrung bis hin zur Zerstörung von Lebenszusammenhängen, schließlich angesichts des Verschwindens von Überlieferung und Erinnerung. Und wie bei einer Blackbox horcht am Ende ihres Lebens auch die Protagonistin des Romans auf die stets noch darin aufgezeichneten Spuren einer verschollenen Welt, versucht den eingegebenen Signalen eine Reaktion und diesen eine Offenbarung zu entlocken, deren Ursprung - die jüdische Schrift - sich in einer Welt ohne Rituale verflüchtigt hat und entzogen bleibt.

\footnotetext{
${ }^{13}$ Schulz‘ Werke, namentlich „Die Zimtläden“ (1933), genießen in ihrer deutschen Übertragung seit längerem eine beträchtliche Popularität. Die aktuellste Übersetzung der „Zimtläden“ durch Doreen Daume stammt von 2008. Jüngstes Zeugnis des Nachwirkens Bruno Schulz‘ bei deutschsprachigen Intellektuellen ist Maxim Billers 2013 vorgelegte Novelle „Im Kopf von Bruno Schulz“.
} 
„Aller Tage Abend“ ist die fiktive Biographie einer Frau, die in fünf hypothetischen Leben angeordnet ist und aus wechselnden Perspektiven erzählt wird. Darin eingeflossen ist die Lebensgeschichte von Erpenbecks mutmaßlich in Lemberg geborener Großmutter, der DDRSchriftstellerin und Journalistin Hedda Zinner, so dass sich auch durch diesen Roman, wenngleich erzählstrategisch anders konzipiert, eine intergenerationelle Spur zieht. Erpenbecks Protagonistin wird im ersten Buch des Romans bzw. in ihrem ersten Leben 1902 als Jüdin in Brody geboren, eine der größten und überwiegend von Juden bewohnten Städte des Kronlandes Galizien und Lodomerien. Die Orte ihrer vier weiteren hypothetischen Leben, d.h. der Bücher II-V sind Wien, Moskau, Ost-Berlin und schließlich Berlin im wiedervereinigten Deutschland. Am Ende jedes Buches steht der (vorläufige) Tod; dazwischen, in den „Intermezzi“, wird im Irrealis die unabwägbare Grenze verhandelt, die zwischen ihm und einem möglichen Weiterleben verläuft, und damit die Frage nach der Kontingenz menschlicher Existenz aufgeworfen, nach der Rolle des bloßen Zufalls oder aber des indirekt oder direkt durch menschliche Entscheidungen und Handlungen herbeigeführten Todes, also des „Gemachten“ von Geschichte. Das offensichtlichste Zeichen, das in Erpenbecks Roman zirkuliert, ist also ein jüdischer und weiblicher Tod.

Damit gestaltet die Autorin ein als prototypisch gedachtes, jüdischmittelosteuropäisches Schicksal, in dem Galizien als Grenzraum zwischen Russland und Österreich und „Armenhaus Europas“14 die europäische Peripherie bezeichnet, den scheinbar unbedeutenden, in Wirklichkeit aber mit Vorzeichen der kommenden europäischen Katastrophe versehene ,Rand‘ des zerfallenden Habsburgerreiches. Von dort aus, von den bereits um die Jahrhundertwende notorischen nationalistischen Konflikten namentlich zwischen Polen und Ukrainern und zahlreichen Pogromen gegen Juden ${ }^{15}$ aus, führt der Weg ins Zentrum der zerstörerischen europäischen Ideologien: österreichischer Faschismus und Antisemitismus, sowjetischer Kommunismus und Stalinismus, ostdeutscher Sozialismus. Für die jüdische Mutter der Protagonistin ist in diesem Zusammenhang die gleichsam seismographische Phantasie aus ihrer Kindheit bezeichnend,

die Welt sei flach wie eine Palatschinke, und gerade sie sei, wie auch die anderen Bewohner der Grenzstadt, an den äußersten Rand dieser Palatschinke gestreut, ein Körnchen Zucker. Hatte sie

\footnotetext{
${ }^{14}$ Vgl. u.a. https://www.herder-institut.de/servicebereiche/verlag/online-shop/detailansicht/ kategorie/tagungenzur-ostmitteleuropaforschung/produkte/galizien.html; 31.01.14. Zu den wirtschaftlichen Verhältnissen in Galizien zwischen 1880 und 1910 s. außerdem Martin Pollack, Kaiser von Amerika. Die große Flucht aus Galizien. Wien 2000, hier v. a. das Kapitel „Das galizische Elend in Ziffern“, 66-80.

${ }^{15}$ Vgl. hierzu u.a. Pollack, Kaiser von Amerika, 78-80.
} 
sich in der Umgebung des Städtchens verirrt, war ihre einzige Angst, dass sie der Grenze zu nahe kam und plötzlich über den Rand fiel. ${ }^{16}$

Dass die Wahrnehmung Brodys als dem Habsburgerreich zugehörendes (Stadt-)Gebiet die eines unheimlichen und bedrohlichen Grenz-Raums ist - man verirrt sich wie ein Fremder darin, läuft gar Gefahr, aus dem Raum herauszufallen, als wären einem seine Koordinaten nicht bekannt -, und eben darin die Wahrnehmung eines Individuums ist, das seinen Platz darin nicht selbstverständlich „hat“, verweist auf die prekäre Situation der galizischen Juden um die Jahrhundertwende. Dies wird auch im Kontrast zu dem Bild deutlich, wie es sich der katholische Vater der Protagonistin von der Donaumonarchie macht, als er, ein K.-u.-k.Beamter, bereits als Einwanderer in Ellis Island an Land gegangen ist: In seinem Blick gerät die Doppelmonarchie unter dem als pater familias idealisierten Kaiser als Raum, in dem sich, „Krieg hin oder her“, schon immer „Völkerschaften“, ja „,von jeher Menschen [...] vermischt und sich, wenn das eine Land zu wenig hergab oder das Leben aus einem anderem (sic) Grund unerträglich geworden war, eine neue Heimstatt gesucht [hatten] “17. Diese dem „Traditionalist[en] Roth“ ${ }^{18}$ teilweise affine, im Emigrationskontext allerdings wenig überzeugende Perspektive muss im Hinblick auf in Buch I dargestellte jüdische Lebenswelt und der Phantasie von der Palatschinke entsprechend ergänzt werden durch eine für den aus Brody stammenden Beschwörer der Donaumonarchie und des „shtetls“ nicht weniger gültige Feststellung von Claudio Magris: „Die Geschichte ist eine Geschichte der Untergänge, der Niederlagen und der Opfer: Exilgeschichte.“19

Eine mythische Bebilderung des ostjüdischen Galizien, die Patina eines „heimatliche[n] Eingepflanztsein[s] in eine Landschaft“ ${ }^{20}$ und in einen Bedeutungszusammenhang jenseits von Geschichte, wie es sich bei Roth, insbesondere in „Hiob“, teilweise bereits gebrochen darstellt $^{21}$, sucht man bei Erpenbeck, bei allen Anklängen an ihn im Hinblick auf Sprache und Milieudarstellung, daher vergebens. Das der prominenten Wirkung talmudischer und chassidischer Kreise in der Grenzstadt entsprechende Einflechten biblischer Erzählungen (etwa

\footnotetext{
${ }^{16}$ Ebd., 37.

${ }^{17}$ Erpenbeck, Aller Tage Abend, 63f.

${ }^{18}$ Claudio Magris, Weit von wo. Verlorene Welt des Ostjudentums. Wien 1974, 22.

${ }^{19}$ Magris, op. cit., 25. In Bezug auf das Motiv der Grenze ist möglicherweise auch eine Affinität zu dessen Gebrauch bei Roth gegeben. Magris bringt es u.a. mit einer „Atmosphäre verzehrender menschlicher Einsamkeit“ in Verbindung; ebd., 36.

${ }^{20}$ Emmanuel Lévinas: „Der Aberglaube des Orts (1961)“, in Günzel, Theorie des Raums, 57-62, hier 60.

${ }^{21}$ Magris nennt „Hiob“ den „ironische(n) Roman eines Intellektuellen, der gerade deshalb die vollkommene Rekonstruktion der Welt und der Humanität des Ostjudentums unternimmt, weil er ihren Verlust und seine nicht wieder rückgängig zu machende Ferne von ihr fühlt.“; Magris, Fern von wo, 113. Vgl. hierzu auch Hans Wagener in seinem „Nachwort“ zu Joseph Roth, Hiob. Roman eines einfachen Mannes. Hg. von Hans Wagener. Stuttgart $2010,213$.
} 
auf die von Lot und den Engeln aus Gen 11,27-31) und jüdischer Legenden, ferner grundlegende jüdische Rituale und Verhaltensrichtlinien ${ }^{22}$ sowie der, allerdings merklich reduzierte, Gebrauch des Jiddischen als Sprache des Familiären und des Intimen („Saj mojchl un fal mir mejne trep nit arunter“; „Maideleh“ ${ }^{23}$ ) - all dies erweist sich im ersten Buch als ein jüdisch-galizischer Gedächtnisraum, in dem die „'vertikale[]` und religiöse Dimension“ bereits Anzeichen ihres bevorstehenden Zerfalls aufweist, die jüdischen Figuren entweder fürchten oder bereits im Begriff sind, aus „einer Welt der transzendenten und transindividuellen Werte“ in die „horizontale Ebene der Geschichte“ einzutreten ${ }^{24}$. Zudem folgt die Darstellung der jüdischen Milieus den für sie kennzeichnenden, aus verschiedenen geschichtswissenschaftlichen und anderen Quellen bekannten sozialen Verwerfungen und Zerklüftungen im ausgehenden 19. Jahrhundert ${ }^{25}$ : Zum einen und vor allem werden hier die (auch in „Hiob“ thematisierten) für die jüdische Lebenswelt (nicht nur) im Galizien der Jahrhundertwende charakteristischen Konfliktlinien nachgezeichnet: eine an „starren [...] Formalismus“ ${ }^{26}$ grenzende Orthodoxie und ärmliche Ghettoexistenz, wie sie sich insbesondre im streng talmud- und thoratreuen Urgroßvater der Protagonistin verkörpern, stehen hier bürgerliche Aufklärung (Haskalah), Ausbruch aus dem Ghetto und Assimilation, wie sie die Mutter der Protagonistin nach und trotz einer orthodoxen Erziehung und einer entsprechenden Vergangenheit betreibt, nahezu unversöhnlich gegenüber - mit den für jüdische Frauen nicht untypischen Folgen: Der nie gelöste Konflikt zwischen Tradition und deren Abstreifen zwecks Aufnahme in die christliche Mehrheitskultur führt bei der Mutter der Protagonistin in die Prostitution (Buch I) ${ }^{27}$, in deren zweiten Leben in Wien (Buch II) in eine Aporie, die sich gleichsam wie eine weibliche Version des bekannten, exemplarisch gewordenen Zitats aus einem Brief Kafkas liest. Wie dieser hält Erpenbeck die leibliche Erfahrung von Ausgrenzung und Zugehörigkeitsnot, die Zerrissenheit des Assimilationsbegehrens fest und unterstreicht sie noch im Verweis auf die Überführung des Somatischen ins Zeichenhafte durch den Prager Schriftsteller:

Der Goj [der Ehemann und k.u.k.-Beamte; A. T.] ist in Ordnung, aber die Tochter hängt nun zwischen den Welten, hängt da und zappelt und kann nicht anders, als sich mit den

\footnotetext{
${ }^{22}$ So öffnet etwa die Mutter der Protagonistin bei deren Tod an einem Sabbat vorschriftsgemäß das Fenster im Sterbezimmer, schüttet alle mit Wasser gefüllten Behälter aus und verhängt alle Spiegel im Haus; vgl. Erpenbeck, Aller Tage Abend, 13.

${ }^{23}$ Ebd., 14; 18.

${ }^{24}$ Magris, Weit von wo, 34; 32.

${ }^{25}$ Verwiesen sei hier u.a. auf Heiko Haumann, Geschichte der Ostjuden. Reinbek bei Hamburg $1999^{5}$.

${ }^{26}$ Magris, Weit von wo, 111.

27 Zur unter den katastrophalen sozioökonomischen Lebensbedingungen in Galizien drastisch ansteigenden jüdischen Prostitution und zum „Handel mit delikatem Fleisch“, die in „Aller Tage Abend“ allerdings nicht ausgestaltet wird, s. u.a. Pollack, Kaiser von Amerika, 53-58.
} 
Füßen von der eigenen Mutter abstoßen, von ihr, deren Züge im Alter so deutlich vom Geschlecht Davids zeugen, dass sie auf der Straße oft angepöbelt wird. ${ }^{28}$

Wie bei Kafkas jungen Juden, die „mit den Hinterbeinchen [...] noch am Judentum des Vaters [klebten] und mit den Vorderbeinchen [...] keinen neuen Boden [fanden]“29, mündet auch bei der Mutter der Protagonistin das Sich-Abstoßen von der eigenen Mutter in die Haltlosigkeit, in ein 'Da-Hängen' und 'Zappeln', Symptom geschichtlich kontingenter Erfahrung, das sich ihrem Körper, ihrer Haut unumkehrbar einschreibt. So heißt es aus der Perspektive ihres Mannes, der dem politischen Elend im Hungerwinter 1919/20 bezeichnenderweise mit dem Studium der „Aufzeichnungen über Erdbeben in der Steiermark“ trotzt $^{30}$ :

Wenn sie aber schläft, setzt er sich gern neben ihr Bett und unternimmt einen weiteren Versuch zu ergründen, was ihm seit einiger Zeit als das größte Rätsel der Menschheitsgeschichte erscheint: wie nämlich Vorgänge, Zustände oder Ereignisse, die allgemeiner Natur sind - zum Beispiel ein Krieg, oder lang andauernder Hunger, oder auch ein Beamtengehalt, das nicht an die rasende Inflation angepasst wird - in ein beliebiges privates Gesicht hineinschlüpfen können. [...] die Abspaltung Ungarns führt vielleicht in dem Gesicht irgendeiner Frau, es kann auch seine eigene sein, zu zerbissenen Lippen. Von ganz weit außen nach ganz weit innen wird also fortwährend übersetzt, nur gibt es dabei für jeden einzelnen Menschen ein eigenes Vokabular, und deshalb hat wohl bisher niemand erkannt, dass es sich dabei um eine Sprache handelt, und zwar um die einzige, die für die ganze Welt und alle Zeit Gültigkeit hat.

Diese 'Übersetzung` von außen nach innen, von Politik und Geschichte in die Intimität des privaten Körpers, der die traumatischen Spuren ersterer als - wie Freuds just in Wien konzipierte Psychoanalyse geltend machte - zutiefst individuelle, humane und gerade darin universelle Sprache lesbar macht, wird ergänzt bzw. fortgeführt durch die ‘Übersetzung` eines verfehlten, gescheiterten Sich-Abstoßens der Mutter in die Tochter-Protagonistin: Bei ihr mündet die dem Körper eingeschriebene Aporie in Buch II im Selbstmord; in Buch III zeitigt sie politische Verblendung - den kurz vor dem Tod in der Verbannung als Irrtum gedeuteten Kampf für den Sowjet-Kommunismus -, verursacht vielleicht gerade durch den allenfalls halbbewussten, eher verdrängten Verlust einer vertikal geordneten und von Transzendenz inspirierten Welt.

Das sichtbare 'jüdische Stigma', auf das hier Bezug genommen wird, steht nun am Anfang einer bemerkenswerten Konstellation, von der aus Erpenbeck den auf Galizien zurückgehenden Gedächtnisraum entwirft; im ersten, von Brody und Joseph Roth inspirierten

\footnotetext{
${ }^{28}$ Erpenbeck, Aller Tage Abend, 98.

${ }^{29}$ Die vollständige Passage aus Kafkas Briefen lautet: „Weg vom Judentum [...] wollten die meisten, die deutsch zu schreiben anfingen, sie wollten es, aber mit den Hinterbeinchen klebten sie noch am Judentum des Vaters und mit den Vorderbeinchen fanden sie keinen neuen Boden.“ Franz Kafka, Briefe 1902-1924. Frankfurt a. M. 1958, 337.

${ }^{30}$ Ebd., hier und für das unmittelbar Folgende 89; 88.
} 
Buch, sind auch schon alle wesentlichen Zeichen angelegt, die im gesamten Roman zirkulieren, Sinn produzieren - und wieder durchkreuzen: Der plötzliche Tod der Protagonistin im ersten Buch - Kind einer „Notzivilehe“, welche die Asymmetrie zwischen Juden und Nichtjuden im Vielvölkerstaat Österreich-Ungarn präzise absteckt ${ }^{31}$ und im Nachhinein als fatale Grenzüberschreitung gedeutet wird -, der den christlichen Vater zur Auswanderung nach Amerika veranlasst, mit der Mitgift seiner jüdischen Frau, aktualisiert einen in der Vergangenheit liegenden, traumatischen Vorfall: Angeführt von dem Bauern Andrej, der als Halbwüchsiger dem Vater der Braut auszuhelfen pflegte, überfällt ein Mob das Familienhaus und erschlägt diesen auf bestialische Weise.

Damit liegt in Erpenbecks Roman nicht nur eine im Vergleich mit „Katzenberge“ unterschiedliche Darstellung Galiziens als multikultureller Grenzraum vor, bei der insbesondere das aus westdeutschen und jüdischen (auch literarischen) Diskursen vielfach bekannte Negativbild vom Polen auffällt: Nicht nur liefert ein kruder Antisemitismus, hier wie auch an anderer Stelle in Buch I ausschließlich polnisch konnotiert ${ }^{32}$, der Händlerwitwe das Motiv dafür, „für das Glück ihrer Tochter das Glück ihrer Tochter“ zu „verkaufen“33 oder, um den Titel des Romans aufzugreifen, aus einer Lebenskatastrophe den Schluss zu ziehen „Am Ende eines Tages, an dem gestorben wurde, ist längst noch nicht aller Tage Abend“ - ein Prinzip, das sich gegen die „existentielle[] Unsicherheit“ in einem jüdischen Sprichwort stemmt: „Vom Morgen bis zum Abend kann die Welt zerstört werden.““34. Auch die Gestaltung des kommunikativen Gedächtnisses fällt dem entsprechend unterschiedlich aus. Stellt sich dieses bei Janesch als intakt, als generationenübergreifend dar und ist daher als (hybride) Zugehörigkeit stiftend konstitutiv für den Roman, so erscheint bei Erpenbeck das galizische Brody als dessen traumatischer Kern. Genau hier gewinnt die Zirkulation bestimmter

\footnotetext{
${ }^{31}$ In der Habsburger Monarchie war die Notzivilehe bei einem kirchenrechtlichen Ehehindernis üblich. In der Regel ging ihr eine „Verweigerung der Eheschließung“ aus konfessionellen Gründen voraus; das entsprechende Eherechtsgesetzt in Österreich stammt vom 25. 5. 1868; s. http://www.enzyklo.de/Begriff/ Notzivilehe; 30.01.14. ${ }^{32}$ Dieser Umstand wird auch kaum , ausgeglichen' durch die kaum differenziertere, allerdings lediglich indirekte und allgemeine Darstellung von Deutsch-Österreichern, insbesondere von den Bewohnern des als Hauptstadt des Antisemitismus verschrienen Wiens in Buch II. Auch im Zusammenhang mit den „Blutnächte[n] von Lemberg“ (Buch II), basierend auf den historischen Auseinandersetzungen zwischen Polen und Ukrainern im November 1918, werden judenfeindliche Ausschreitungen mit ersteren in Verbindung gebracht. Überdies sind die Namen der ausdrücklich als Polen identifizierten Mörder falsch geschrieben („Andrej“ statt „Andrzej“; „Krystof“ statt „Krzysztof“. Schließlich ist im Zusammenhang mit einem Brief der Protagonistin an die Mutter, den erstere in Buch III mit dem Vermerk „Evakuiert nach dem Osten“ zurück erhält, womit der reale, doch unbekannte Ort der Vernichtung ersetzt wird durch einen imaginären Raum, festzustellen, dass in „Osten“ als Kürzel für den nationalsozialistischen Massenmord an den Juden Polen nicht als konkret Geographisches, sondern als „Wortvorstellung dieses Ostens“ mitschwingt; Erpenbeck, Aller Tage Abend, 206. Zum Polenbild in der deutschsprachigen Literatur vgl. Barbara Breysach, Schauplatz und Gedächtnisraum Polen. Die Vernichtung der Juden in der deutschen und polnischen Literatur. Göttingen 2005, 184.
}

33 Ebd., 52.

${ }^{34}$ Magris, Weit von wo, 134. 
„tokens“ an Bedeutung: Sind es bei Janesch das Motiv der galizischen Erde und des großväterlichen Wortes bzw. der ,galizischen Geschichten‘, die einen intergenerationellen Gedächtnisraum ungebrochen aufrechterhalten, so zeigt sich dieser bei Erpenbeck nicht nur als gebrochen, sondern als abgebrochen: der Verdrängung, der Vernichtung, dem Vergessen anheimfallend und damit der Funktion einer Sinnstiftung verlustig gehend. So leitet der traumatische Mord am Juden bereits die unumkehrbare Verfluchung und Verstoßung der Tochter durch den jüdischen Großvater ein, als diese auf Betreiben der Mutter mit dem K.-u.k.-Beamten, einem „Goj“, verheiratet wird; damit ist das Ende jedweder Art ,großväterlichen Wortes‘, d.h. eines intergenerationellen Gedächtnisses, das in Bezug auf seine eigene Tochter noch gegeben war, besiegelt. Auf einer anderen Ebene wird dieser Vorgang des NichtErzählens von der Mutter selbst vollzogen, indem sie die Tochter über den ermordeten Vater zunächst belügt - er sei fortgegangen, womöglich ausgewandert -, dann einen Satz sagt, den die Tochter wiederum ihrer eigenen Tochter verschweigt: „Deinen Vater haben die Polen umgebracht.“ “35 Auch das in Buch I eingeführte, leitmotivische Bild des Briefs, das „,[I]n der sonnigen Stille eines Sabbats [...] aus einer sich öffnenden Hand in eine Hand [fällt], die jemand aufhält“ - mit der „Heiligkeit des Lebens“ eine Gedankenkonstruktion, über welche die Großmutter einst mit Blick auf die Sabbatregeln im Talmud mit deren Vater zu diskutieren pflegte - und das Bild des Stürzens zeigt in Buch IV das fatale Schwinden der vom kommunikativen Gedächtnis gestifteten Bezüge an:

\footnotetext{
Aber der eine muss den Brief abgeben wollen und der andre ihn nehmen, hatte sie der Großmutter entgegnet.

So ist es.

Und der Wille ist keine Arbeit?

Wenn ihr jetzt einfiele, was die Großmutter auf diese Frage geantwortet hat, würde alles noch einmal gut.

Aber es fällt ihr nicht ein.

Sie stürzt. ${ }^{36}$
}

Der unumkehrbare (Ab-)Bruch in der Überlieferung ist in Buch V zu besichtigen. Er spiegelt sich in weiteren Merk-Malen/“tokens“, die einen prominenten Platz im halb assimilierten jüdisch-galizischen Haus haben und sich als semantisch aufgeladene Bedeutungszeichen ebenfalls durch den ganzen Roman ziehen: die Gesammelten Werke Goethes, deren neunter Band beim mörderischen Angriff Andrejs beschädigt wird, und eine kunstvoll gearbeitete Standuhr. Als Objekte einer bedrohten Zugehörigkeit und eines grausam zunichtegemachten

\footnotetext{
${ }^{35}$ Ebd., hier und für das unmittelbar Folgende 40; 52.

${ }^{36}$ Ebd., 223 und in diesem Zusammenhang ferner 52f.; 223.
} 
bildungs(-inter-)kulturellen Begehrens fallen sie noch vor der Deportation der Großmutter in die Hände von österreichischen „Reichsbürgern“. Ebendiese Merk-Male - Goethe und die Standuhr - wird in Buch V der nun erwachsene Sohn der auf den Tod wartenden Protagonistin, Zeitgenosse einer spektakulären Grenzaufhebung inmitten Europas, selbst herkommend aus einem „untergegangenen Land“, der DDR, und eben darum ein freier Fremder in der alles dominierenden westlichen „sogenannten Moderne“37, bei einem Antiquar in Wien nicht wiederfinden, sondern ahnungslos registrieren. Denn der unwissende Sohn erkennt sie nicht als das, was sie sind, ebenso wenig wie er erkennen kann, woher sich die Lebensspur seiner Mutter seiner Existenz eingeschrieben hat, einer Frau, die selbst um die Sprache ihres Gedächtnisses ringt. „Weißt du, sagt sie, ich habe Angst, dass alles verlorengeht - dass die Spur verlorengeht. Welche Spur, fragt der Sohn. Ich weiß nicht mehr, woher und wohin.“38 Inmitten einer Aufreihung von Sätzen, die mit „Ich weiß nicht“ beginnen und die Grenze zum nahenden Tod anzeigen, fällt dann der entscheidende Satz: „Ich weiß nicht, wo ich zu Haus war.“ Die Erinnerungsspur, die sich hier ausschließlich im Nicht-Wissen vermittelt, kann so „weder Erinnerungscharakter, noch Vergangenheitsqualität“39 annehmen. Mit Aleida Assmann zu sprechen könnte man auch sagen: Nicht nur versagt hier das „Funktionsgedächtnis“, indem Erinnerung nicht aktualisiert wird, folglich auch kein „Prozess der Sinngebung und Identitätsbildung“ sich einstellt; auch das „Speichergedächtnis“, das „potentiell verfügbare Erinnerungsmaterial“, ist buchstäblich unauffindbar. ${ }^{40}$ Von hier aus kommt dem Sohn die paradoxe Freiheit zu, nichts „loswerden“, aber auch nichts „verlieren“ zu können; als letztes Glied in der Kette ist er Insasse eines Echoraums, indem nurmehr unlesbare Zeichen zirkulieren:

Es hat nicht einmal ein Menschenleben gedauert, bis ihn, den Nachfahren einer Wienerin, diese Stadt nichts mehr angeht. Nicht einmal ein Menschenleben, bis Herkunft und Heimat zweierlei sind. Frei ist er, doppelt frei, in seinem Innern trägt er als ein großes schwarzes Land all die Geschichten, die seine Mutter ihm nicht erzählt oder verschwiegen hat, mit sich herum, trägt vielleicht sogar diejenigen Geschichten, die nicht einmal seine Mutter wusste oder in Erfahrung gebracht hat, mit sich herum, kann sie nicht loswerden, aber sie auch nicht verlieren, weil er sie gar nicht kennt, weil all das in ihm begraben ist, weil er mit Innenräumen, die ihm nicht gehören, schon aus seiner Mutter geschlüpft ist und sein eigenes Inneres nicht anschauen kann. ${ }^{41}$

\footnotetext{
${ }^{37}$ Ebd., 264.

${ }^{38}$ Ebd., hier und für das im unmittelbar Folgenden Zitierte 278; 279.

${ }^{39}$ Mario Erdheim: „Das Unbewusste in der Kultur. Erinnern und Verdrängen als Themen der

Kulturwissenschaften“, in: Jaeger/Rüsen, Handbuch der Kulturwissenschaften Bd. 3, 92- 108, hier 101.

${ }^{40}$ Emil Angehrn. „Kultur und Geschichte - Historizität der Kultur und kulturelles Gedächtnis“, in:

Jaeger/Liebsch, Handbuch der Kulturwissenschaften Bd. 1, 385-400, hier 393.

${ }^{41}$ Erpenbeck, Aller Tage Abend, 267.
} 
Geteilte Erinnerung - im doppelten Sinne und vielleicht auch in exemplarischer Weise zeigt sich dies in den Romanen von Sabrina Janesch und Jenny Erpenbeck. Als historischer Ort der Verfolgung, Vertreibung und Ermordung von Polen durch Ukrainer und als Referenzpunkt eines mythisch angehauchten, dabei aus der generationalen Distanz auch teils ironisch gebrochener Sehnsuchts- und 'Ursprungsort' im kommunikativen Gedächtnis erscheint Galizien bei der deutsch-polnischen Schriftstellerin; in galizischen „tokens“, in der Erde und im Wort, in den Erzählungen des Großvaters, zirkuliert dieser Gedächtnisort noch bis in die dritte Generation. Ganz anders Erpenbeck: Hier bildet Galizien zunächst einen prekären Grenzraum, der aus einer nahezu ausschließlich jüdischen Perspektive geschildert wird und aller Aura entbehrt, ein Raum auch, in dem Polen nicht als die einzigen, aber ausschließlich als Aggressoren gekennzeichnet werden, und der sich im Verlauf dreier Generationen zu einem dunklen, blinden Fleck, zu einer nicht mehr auffindbaren Spur im kommunikativen Gedächtnis verflüchtigt - nur noch an nicht mehr identifizierbaren Objekten haftend, die nicht länger zwischen den Generationen zirkulieren. Dass dies nicht nur Resultat komplexer Assimilationsprozesse infolge von antisemitischer Anfeindung und Ausgrenzung, sondern auch und vor allem des nationalsozialistischen Massenmordes an den osteuropäischen Juden ist, rückt die Darstellung des (jüdischen) 'Ursprungs' strukturell in die Nähe zu Jacques Derridas dekonstruktiven Konzept der Schrift, hier aufgrund des unterschiedlich gearteten Kontextes und Narrativs jedoch gänzlich anders als bei Janesch: Als literarischer Text, der sich der vernichteten Judenheiten auch „stellvertretend als einer Gedächtnisschrift annimmt“, führt „Aller Tage Abend“ ganz am Ende in der Figur des weinenden Sohnes der jüdisch-galizischen Schriftstellerin-Protagonistin den Nachvollzug einer fragmentarischen, beinah unentzifferbar gewordenen Schrift - ihrer Biographie - als Erfahrung einer radikalen Verwaisung vor Augen: Was bleibt, ist eine Spur, die auf ein „Original ohne Ursprung“ verweist, auf die “Anwesenheit einer Abwesenheit ohne repräsentative Dignität“ und die „'Verlorenheit‘ des Gemeinten in der Schrift“. ${ }^{42}$

\section{Literatur}

Angehrn, Emil (2011): „Kultur und Geschichte - Historizität der Kultur und kulturelles Gedächtnis“, in: Jaeger/Liebsch, Handbuch der Kulturwissenschaften Bd. 1. Stuttgart, 385-400.

Assmann, Aleida $\left(2008^{2}\right)$ : Einführung in die Kulturwissenschaft. Grundbegriffe, Themen, Fragestellungen. Berlin.

Barbara Breysach (2005), Schauplatz und Gedächtnisraum Polen. Die Vernichtung der Juden in der deutschen und polnischen Literatur. Göttingen.

\footnotetext{
${ }^{42}$ Vgl. Breysach: Schauplatz und Gedächtnisraum Polen, 44.
} 
Erdheim, Mario (2011): „Das Unbewusste in der Kultur. Erinnern und Verdrängen als Themen der Kulturwissenschaften“, in: Jaeger, Friedrich und Jörn Rüsen (Hrsg.), Handbuch der Kulturwissenschaften Band 3: Themen und Tendenzen. Stuttgart und Weimar, 92- 108.

Galizien und Schlesien. Zwangsumsiedlung und Vertreibung in den vierziger Jahren. Galizien“, in: http://www.sabrinajanesch.de/werke/katzenberge/galizien-und-schlesien/; 31.10 .13

Gnauck, Gerhard: „Männer und Frauen, grausam mit Äxten zerhackt“, in: Die Welt, 26.06.2013; http://www.welt.de/geschichte/zweiter-weltkrieg/ article117455158/Maenner-und-Frauengrausam-mit-Aexten-zerhackt.html; 08.01.2014.

Granach, Alexander: Da geht ein Mensch. Roman eines Lebens. München/Zürich 1990 (hier zit. nach Haumann: $\left.1999^{5}\right)$.

Kafka, Franz (1958), Briefe 1902-1924. Frankfurt a. M.

Kimmich, Dorothee und Schamma Schahadat (2012), Einleitung zu dies. (Hrsg.): Kulturen in Bewegung. Beiträge zur Theorie und Praxis der Transkulturalität. Bielefeld, 7-21.

„Der Konflikt mit den Ukrainern in Wolhynien und Ostgalizien“ (2009), in: Atlas Zwangsumsiedlung, Flucht und Vertreibung. Ostmitteleuropa 1939-1959. Warszawa, 71-74.

Emmanuel Lévinas: „Der Aberglaube des Orts (1961)“ (2013), in: Stephan Günzel, Texte zur Theorie des Raums. Stuttgart, 57-62.

Claudio Magris (1974), Weit von wo. Verlorene Welt des Ostjudentums. Wien.

Nora, Pierre, „Die Gedächtnisorte (1984)“ (2013), in: Stephan Günzel, Texte zur Theorie des Raums. Stuttgart, 84-89.

Martin Pollack (2000), Kaiser von Amerika. Die große Flucht aus Galizien. Wien.

Ricoeur, Paul: „Poetik und Symbolik - Erfahrung, die zur Sprache kommt“ (2011), in: Friedrich Jaeger und Burkhard Liebsch (Hrsg.), Handbuch der Kulturwissenschaften Band 1: Grundlagen und Schlüsselbegriffe. Stuttgart und Weimar, 93-105.

Schulz, Bruno (2008): Die Zimtläden. Übersetzt von Doreen Daume. München.

Wagener, Hans (2010): Nachwort zu Joseph Roth, Hiob. Roman eines einfachen Mannes. Hg. von Hans Wagener. Stuttgart.

Welsch, Wolfgang, „Was ist eigentlich Transkulturalität?“ (2012), in: Kimmich, Dorothee und Schamma Schahadat (Hrsg.): Kulturen in Bewegung. Beiträge zur Theorie und Praxis der Transkulturalität. Bielefeld, 25-40.

https://www.herder-institut.de/servicebereiche/verlag/online-shop/detailansicht/kategorie/tagungenzur-ostmitteleuropaforschung/produkte/galizien.html; 31.01.14.

http://www.enzyklo.de/Begriff/ Notzivilehe; 30.01.14.

http://de.wikipedia.org/wiki/Hedda_Zinnerhttp://

www.kuenstlerkolonie-berlin.de/bewohner/zinner.htm\#zinner 